\title{
Report on 'Innovations in healthcare, patient safety and simulation": Fifth Annual Simulation Conference, Homerton University Hospital Foundation Trust, 10 December 2015
}

\section{Rebecca Szekely, ${ }^{a}$ Mhairi Jhugursing ${ }^{b}$ and Chris Sadler ${ }^{c, *}$}

${ }^{a}$ Whipps Cross University Hospital, London E11 1NR, UK; ${ }^{b}$ The Homerton University Hospital NHS Foundation Trust, London E9 6SR, UK; ${ }^{c}$ Director of Simulation UCLPartners \& Barts Health NHS Trust, Royal London Hospital, Whitechapel E1 1BB, UK ${ }^{*}$ Corresponding author at: Director of Simulation UCLPartners \& Barts Health NHS Trust, Royal London Hospital, Whitechapel E11BB,UK. Email: chris.sadler@bartshealth.nhs.uk

Date accepted for publication: 15 June 2016

\section{Abstract}

The Homerton University Hospital NHS foundation Trust delivered its 5th annual simulation conference on 10th December 2015. A review of the conference is provided in this report.

\section{Introduction}

The Homerton University Hospital NHS foundation Trust delivered its 5th annual simulation conference on 10 December 2015. The title of the conference was 'Innovations in healthcare, patient safety and simulation': a perfect description of the Homerton's ethos.

\section{Session 1: technology and human factors}

Dr Helgi Johannsson, Consultant Anaesthetist, Imperial College Healthcare, London

Web2.0: social media and the changing face of healthcare ${ }^{1}$ Dr Johannsson opened the proceedings by announcing that there would be 'live tweeting throughout the day' and the audience responded promptly by posting tweets displayed on screen. A show of hands from the delegates revealed that almost everybody had an account with either Twitter or Facebook.

He went on to explain that Web 2.0 describes World Wide Web sites that emphasize user-generated content, usability, and interoperability. Social media is a two-way process that has already changed the way that we as health care providers interact with each other, our institutions, our patients and the wider world. He provided examples of how social media is being utilized in health care:

(1) Collaboration: @OG SQILL (OroGastric Surgery Quality Improvement Alliance). This is a Twitter site for sharing best practice in upper gastrointestinal surgery

(2) Sharing knowledge. Social media and critical care conference (http://www.smacc.net.au) delegates play a key role in the conference and can ask questions to speakers from afar using social media. Ongoing online conversations allow delegates to enhance their learning experience.

(3) Learning from adverse events. Dr Johannsson gave a personal example of learning from wrong site surgery by posting 'Wrong Footed' using Storify (available at https://storify.com/traumagasdoc/wrongfooted,

accessed 6 June 2016). This received 22,000 hits and he argued this reached a much bigger audience than a journal article might and allowed sharing of knowledge with largely positive feedback.

(4) Interacting with patients, public, media and management. Dr Johannsson gave examples of how social media might be used for booking patient appointments, which could be quicker and more reliable than traditional mail. He emphasized that we could learn from other industries (e.g. British Airways) that already receive complaints via live feeds. He gave examples of how medical advice could be given via social media (e.g. Christian Jessen, presenter of Channel 4's Embarrassing Bodies TV programme).

(5) Medical organizations. Many medical institutions have signed up to the use of social media to interact with 
their members and the public including: @TheBMA, @GMCUK, @RCoANews, @AAGBI. Dr Johannsson suggested that NHS Trusts could do more with social media to interact with patients.

(6) Influencing press campaigns. The junior doctors' strike was given as an example where doctors could exert an influence on press campaigns

Dr Johannsson finished up by reminding all of us that the General Medical Council had produced guidance in Good Medical Practice on the use of social media, which he recommended that we all read before we start posting. ${ }^{2,3}$

\section{Lt Col Claire Park MBE, Kings College Hospital, London's Air Ambulalance, London}

Team resource management: lessons from military and pre-hospital care ${ }^{4}$

Lt Colonel Park shared her considerable knowledge of human factors gained from working in medical emergency response teams in Iraq and Afghanistan. Many of the concepts explored should be embedded in the training of NHS teams. Human factors issues such as bandwidth, situation awareness, leadership, decision making, command gradients, confirmation bias and recency were covered with coping strategies provided to reduce the chances of falling into human error traps. The term 'the deadly set' was described as that situation where you believe what you are told and you join that thought process. This happens in NHS health care and has direct relevance to simulation training. If we brief an anaesthetist about to go into a scenario with the statement 'could you come to labour room 2 please? The epidural has just been topped up and the lady is hypotensive', we have potentially given a 'deadly set' making the anaesthetist think the hypotension is related to the epidural rather than the concealed antepartum haemorrhage; a great way of demonstrating this principle. We also heard that it is important in simulations to run them as true to life as possible, opening kit that is used infrequently and using it. Regression says that we tend to go back to what we are familiar with in emergency situations so we need to be familiar with emergency kit and drugs. With regard to transfer of training, if you do not do things completely in simulation then this tends to be the case in real life as well. There were good parallels around team briefing and the use of the WHO checklist but the use of SitReps (review of time, blood loss, clotting and surgical process) every 15 min might enhance team situation awareness and decision making in NHS surgical emergencies.

\section{Session 2: surgical innovations}

Mr Sanjay Purkayastha: Imperial College, London

\section{Usher: patient support across the bariatric surgical pathway $^{5}$}

Mr Purkayastha introduced us to mobile health, the use of mobile devices to support the practice of medicine and public health. His research suggests that $80 \%$ of his patient group have smart phones and there was underuse of this technology in health care. Usher is a smart phone-based platform for patient support and education for bariatric surgical pathways. This was developed using a collaborative approach with members from every part of the pathway. The app gives information to patients and also tests their understanding of this information. The developers have access to these test results, which they can review to support each patient's needs. Patients can input their calorific intake and upload pictures of their meals, which can be reviewed by the bariatric team. A video of the app was shown, which features a lot of animation and the patients have given excellent feedback. We will be interested to see the results of a randomized controlled trial on the use of Usher.

\section{Mr Andre Chow: Touch Surgery (www.touchsurgery. com)}

Teaching millennial surgeons: challenges and solutions ${ }^{6}$ Surgery has become more complex. Technological advances produce more specialized equipment. Higher risk patients are taken on and the patients have greater expectations of health care. Surgeons therefore require an increasing breadth of knowledge and skills but they also need new ways of training because operative time has decreased. Surgeons have also changed in that Generation $\mathrm{Z}$ wants to learn on demand. Touch surgery provides a solution in providing training that is digital, mobile and interactive. It is an internet-based visual platform developed in collaboration with digital film makers who have worked with Pixar. They currently provide over 30,000 hours of simulation per month for a range of surgical procedures and clinical skills. The programmes focus on cognitive decision making, which is thought to account for as much as $75 \%$ of surgical success. In the United States, this has been incorporated into residency programmes as a validated training tool allowing digital certification. In the United Kingdom, there is no requirement for surgeons to practice on a simulator before they can practice on a patient. Surely it is time this changed? 


\section{Emma Jones: University of Leicester}

SIQINS: strengthening implementation of quality improvement interventions and methods in surgery ${ }^{7}$

Quality improvement (QI) intervention designed to improve patient care in surgery is a rapidly expanding field. However, Ms Jones provided evidence that published reports are poor at describing the interventions fully, including what was done and how it was delivered. This makes it difficult for other health care professionals to translate the findings of QI research into practice in their own institutions. Ms Jones introduced us to two checklists that prompt authors to describe interventions to a standard that would allow their replication: TIDieR (template for intervention, description and replication) and SQUIRE (Standards for Quality Improvement Reporting Excellence). She plans to look at why authors find it difficult to describe their QI work in full by using qualitative methods from interview studies. This will include reporting how human factor-based QI interventions in areas such as leadership have improved outcomes.

\section{Session 3: simulation and patient safety \\ Dr Helen Parker and Dr Nick Walsh, Newham University Hospital, London}

\section{Simulation for Acute Psychiatric Emergencies (APE) ${ }^{8}$}

Dr Parker and Dr Walsh designed and set up an innovative simulation experience. The APE (Acute Psychiatric Emergencies) course is both a multidisciplinary and an inter-trust collaboration. A\&E staff learn practical aspects of patient management for acute psychiatric emergencies with community psychiatric staff, and this promotes better team working with a greater appreciation of each other's roles. Scenarios included acute psychosis, delirium tremens and assessing risk of suicide. De-escalation techniques such as tone of speech, body language and body proximity seemed particularly transferable to generic practice. Both open simulation (group in a circle with simulation occurring in the middle) and closed simulation (participants and observers separated by mirror) set-up techniques were used. Feedback has been positive with a recurring comment that before the APE course participants had little training on the acute mental health issues raised. This reiterated the value of the course. The continued development of the APE course is an important step in the field of psychiatric simulation.

\section{Dr Lysa Owen, Clinical Skills Centre, University of Dundee}

From fairies to SimMan: Tolkien and realism in simulation ${ }^{9}$ Dr Owen's title was intriguing: fairies, hobbits and simulation? Simulation, like The Lord of the Rings is a type of fantasy whereby the participants must engage, believe and immerse themselves in the story around them. Dr Owen's research focuses on what determines a participants' engagement in simulation. Group interviews of medical students and doctors of all grades were conducted and analysed. Realism was a recurring theme. Deickmann et al. ${ }^{10}$ described realism as having physical, semantic and phenomenological components. There is attention to detail on the physical simulation environment, but more challenging is semantic and phenomenological realism, where the progress of the scenario is believable and engenders similar emotions as the real situation. It is these two latter components of realism that the interviewed groups identified as important in the motivation to engage in simulation. Dr Owen beautifully illustrated this with a montage of fictional clips, some very believable and some sitting uneasily in their environment. This insight into key components of realism in simulation is an area we will certainly be putting more emphasis on when designing scenarios.

\section{Professor Sheila Adam, Homerton University Hospital NHS Foundation Trust}

Advancing patient safety: the role of hospital IT (and the Chief Nurse) ${ }^{11}$

Professor Adam discussed the introduction of the electronic patient record (EPR) system at the Homerton Hospital and its role in promoting patient safety. In 2014, the National Reporting and Learning Service showed that $23 \%$ of deaths in hospitals were associated with failure to recognise or act on deterioration of the sick patient. ${ }^{12}$ Risk is present in all health care systems with increasing patient comorbidities, workload demands and process/system demands. The EPR system provides reliability and stability in process. It allows patient data to be safely stored and securely shared among health care professionals in primary and secondary settings. Targeted alerts and guidance, e.g. allergies, early warning scores, sepsis and falls can be displayed in one place on a patient dashboard for easy viewing, facilitating recognition and early management of ill patients. The continued refinement of the EPR system by the information technology team and the patient safety team make a significant contribution to safety and quality of patient care at the Homerton Hospital.

\section{Session 4: quality improvement}

\section{Dr Chris Kowalski, South London \& Maudsley NHS Foundation Trust}

Interprofessional simulation to improve collaborative care for young people with physical and mental health needs ${ }^{13}$ Dr Chris Kowalski kicked off the last session of the afternoon speaking about 'Interprofessional simulation for young 
people with physical and mental health need'. The course had been developed at the Maudsley's simulation centre, the UK's first focusing on mental health, although priding itself that its courses can be delivered at any location. Scenarios included management of anorexia, deliberate self harm and psychosis. Despite diagnoses of mental health problems in young people increasing (the diagnosis of depression having doubled in 15/16 year olds in the last decade), staff confidence in dealing with young people with mental health problems is low. The 1 day course, which has 12 participants covering the multidisciplinary team, includes six scenarios and has received excellent feedback. Focus groups have revealed that there is better awareness of the issues, improved handover and earlier involvement of colleagues. The course continues to run and it is aimed to roll out to other sites.

\section{Dr Martin Kuper, Homerton University Hospital NHS Foundation Trust}

\section{Enhanced recovery pathway improvement ${ }^{14}$}

Since guidelines for the implementation of enhanced recovery protocols were released in 2009, ${ }^{15}$ together with 'a better journey for patients and a better deal for the NHS' in $2010,{ }^{16}$ the practice has become widespread, particularly in colorectal and musculoskeletal surgery. Dr Kuper, a member of the Enhanced Recovery Partnership Publication Working Group, gave us an update on 'enhanced recovery after surgery'. Essentially, enhanced recovery is a success, saving the NHS $£ 2.3$ billion by reducing hospital stay, partly by simple protocol-led measures such as encouraging patients to wear their own clothes and reducing intravenous fluid administration, making patients more mobile. He explained that new pathways can be developed through simulation, and do not need long and costly randomized controlled trials, so patients and the NHS can see the benefits earlier.

\section{Dr Maris Chazapis: Anaesthetist, UCL/UCLH Surgical Outcomes Research Centre (SOuRCe), University College Hospital, London}

\section{Improvement science. What is it? How is it done? ${ }^{17}$}

The final offering of the day, from Dr Chazapis, was a journey through improvement science, from its origins to practical everyday application. Even though 'quality improvement' has made a recent insurgence into our daily lives, it started back in 1928 with Walter Shewhart, with the advent of the PDCA (plan, do, check, act) cycle later revised by Deming to the commonly known PDSA (plan do, study, act) cycle. A commonly held myth was quashed: is QI the same as audit? The simple answer is no; audit is what is happening right now and QI is what can happen, focusing on the six dimensions of quality coined by Donal Berwick: safe, effective, patient centred, timely, efficient and equitable. Dr Chazapis has helped to develop excellent educational resources available at www.prism-ed.com to support anyone involved in a QI project.

This thought provoking conference delivered on the themes given in its title: Innovations in health care, patient safety and simulation. We were introduced to a range of innovative screen-based educational tools that are already making a difference to the training of our workforce and the education and safety of our patients. It was particularly pleasing to see that the conference organizers kept the content current with inclusion of informative sessions on quality improvement. It was also refreshing to see that simulation spans so many areas of health care and is helping to break down the barriers between hospital and community with courses like APE.

\section{Conflict of interest}

No conflicts of interest have been declared.

\section{References}

1. Johannsson H. Web 2.0: social media and the changing face of healthcare (abstract). J Surg Simul 2016. doi: 10.1102/ 2051-7726.2016.A001.

2. General Medical Council. Good medical practice. London: GMC; 2013. Available at http://www.gmc-uk.org/static/docu ments/content/GMP_pdf. Accessed June 6, 2016.

3. General Medical Council. Doctors' use of social media. London: GMC; 2013. Available at http://www.gmc-uk. org/static/documents/content/Doctors_use_of_social_media. pdf. Accessed June 6, 2016.

4. Park C. Team resource management - lessons from military and pre-hospital care (abstract). J Surg Simul 2016. doi: 10. 1102/2051-7726.2016.A002.

5. Purkayastha S. Usher: patient support across the bariatric surgical pathway (abstract). J Surg Simul 2016. doi: 10. 1102/2051-7726.2016.A003.

6. Chow A. Teaching millennial surgeons: challenges and solutions (abstract). J Surg Simul 2016. doi: 10.1102/2051-7726. 2016.A004.

7. Jones E. SIQINS - strengthening implementation of quality improvement interventions and methods in surgery (abstract). J Surg Simul 2016. doi: 10.1102/2051-7726.2016.A005.

8. Parker H, Walsh N. Simulation for acute psychiatric emergencies (APE) (abstract). J Surg Simul 2016. doi: 10. 1102/2051-7726.2016.A006. 
9. Owen LE. From fairies to SimMan: Tolkein and realism in simulation. J Surg Simul 2016; 3: 8-12. doi: 10. 1102/2051-7726.2016.A007.

10. Dieckmann P, Gaba D, Rall M. Deepening the theoretical foundations of patient simulation as social practice. Simul Healthc 2007. 2: 183-193. doi 10.1097/SIH.0b013e3180f637f5.

11. Adam S. Advancing patient safety - the role of hospital IT (and the chief nurse) (abstract). J Surg Simul 2016. doi: 10. 1102/2051-7726.2016.A008.

12. Donaldson LJ, Panesar SS, Darzi A. Patient-safety-related hospital deaths in England: thematic analysis of incidents reported to a national database, 2010-2012. PLoS Med 2014; 11: e1001667. doi: 10.1371/journal.pmed.1001667.

13. Kowalski C, Attoe C, Cross S. Inter-professional simulation to improve collaborative care for young people with physical and mental health needs (abstract). J Surg Simul 2016. doi: 10. 1102/2051-7726.2016.A009.

14. Kuper M. Enhanced recovery pathway improvement (abstract). J Surg Simul 2016. doi: 10.1102/2051-7726.2016. A010.

15. Khan S, Gatt M, Horgan A, Anderson I, MacFie J. Guidelines for implementation of enhanced recovery protocols. London: Association of Surgeons of Great Britain and Ireland; 2009.

16. NHS Improvement. Fulfilling the potential: a better journey for patients and a better deal for the NHS. NHS Enhanced Recovery Partnership 2012.

17. Chazapis M. Improvement science - what is it? How is it done (abstract)? J Surg Simul 2016. doi: 10.1102/2051-7726. 2016.A011. 